\title{
Developing and selecting interventions for translating knowledge to action
}

\author{
Michel Wensing PhD, Marije Bosch PhD, Richard Grol PhD
}

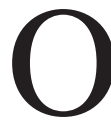
nce knowledge has been adapted to local context and the barriers to and facilitators of use of knowledge have been identified, ${ }^{1}$ we must select, tailor and implement interventions for the uptake of knowledge. ${ }^{2}$ This article provides an approach to selecting interventions for knowledge translation.

How would we select interventions to translate the knowledge from practice guidelines and research into practice? For example, major variations in the treatment of chronic heart failure have been found repeatedly. In one survey, use of $\beta$-blockers in primary care ranged from $10 \%$ to $50 \%$ between countries, and use of angiotensin-converting enzyme (ACE) inhibitors ranged from $50 \%$ to $75 \%{ }^{3}$ Differences in the recommendations of national guidelines were not sufficient to explain this variation. ${ }^{4}$ Comorbidity explained some of the variation, but $14 \%$ of prescriptions were related to characteristics of patients that were not aligned with evidence. ${ }^{5}$ A study of barriers to adherence to guidelines related to heart failure showed that many family physicians found it difficult to change treatment initiated by a cardiologist. Titrating the dosage of ACE inhibitors and initiating ACE inhibitors in patients who were already using a diuretic or were stable on their current medication were perceived as barriers. ${ }^{6}$

Interventions to facilitate the uptake of research could include training for physicians (e.g., to learn about titrating the dosage of ACE inhibitors), the use of opinion leaders to influence patterns of prescribing by cardiologists, or the provision of financial incentives to physicians for each patient with heart failure who is treated according to guidelines. Alternatively, educators could better inform patients and their families about appropriate care in the hope that they will ask for such treatment in future consultations with clinicians. Ideally, the selection of an intervention for knowledge translation should be guided by research-based evidence of the effectiveness and efficiency of the various interventions. However, this evidence cannot explicitly guide our decisions in all situations and circumstances. Therefore, in addition to science, we will need some artistry to choose or design the intervention. ${ }^{7}$

A review of all of the evidence on developing and selecting interventions is beyond the scope of this paper; instead we will summarize available systematic reviews. ${ }^{7.8}$ Many interventions for knowledge translation have not been well evaluated with rigorous studies. For interventions that have been evaluated, evidence suggests that their impact is variable and, on average, the effect size is moderate.

\section{Key points}

- Interventions for knowledge translation need to be tailored to specific barriers to change.

- The effectiveness of tailored interventions varies substantially, partly because methods of tailoring vary widely across studies.

- Multicomponent interventions for knowledge translation are not consistently effective either, partly because the definition of a multicomponent intervention is not clear.

- Research-based evidence can provide some guidance but cannot show decisively which intervention is most appropriate.

- Selecting interventions is an "art" that can be supported by using structured methods to choose objectives, identify barriers to change and link interventions to these barriers.

Several conclusions can be drawn from the literature. Available research focuses mainly on professional interventions, such as educational programs, feedback and reminders. The methodological quality is variable but moderate overall. The overall absolute change in professional performance is usually not more than $10 \%$ on selected outcomes, but this finding can be clinically or economically relevant.

Passive educational interventions, such as written guidelines, lectures and conferences, are unlikely to change behaviour if used alone. Active educational interventions, such as outreach-based visits, are more likely to induce change. Materials or websites for active self-study (e.g., for distance-based learning) can be effective.

Professional interventions that bring information close to the point of decision-making, such as reminders and decisionsupport tools, are likely to be effective. Patient-directed interventions, such as preconsultation questionnaires or decision aids, can support improvement of quality in some cases, but insight into their effects on quality of care is limited. Organizational interventions, such as revision of professional roles and enhanced teamwork, can influence clinical outcomes and efficiency in some cases, but their impact on uptake of knowledge is unclear.

From the Scientific Institute for Quality of Healthcare (Wensing, Bosch Grol), Radboud University Nijmegen Medical Centre, Nijmegen, the Netherlands; Heidelberg University Hospital (Wensing), Department of General Practice and Health Services Research, Heidelberg, Germany

CMAJ 2009. DOI:10.1503/cmaj.081233 
Financial interventions for patients or professionals influence how many contacts and procedures take place, which may be relevant for the improvement of quality (e.g., by increasing the volume of preventive services rendered). Their effect on the appropriateness of clinical decisions and patterns of practice is less clear. Moreover, evidence for the sustainability of these interventions is limited.

Experts in implementation suggest that a structured approach at various levels is needed to address professionals, patients, teams, organizations and systems of health care. ${ }^{8}$ Structured approaches to planning for change have been developed in various scientific disciplines and include mapping of interventions, marketing, small-group quality improvement, management of change, organizational development and assessment of technology used in health care. ${ }^{9}$ Whether these structured approaches result in better uptake of knowledge and which of their components are most relevant remain unproven. The models of planning for change propose approximately the same steps or stages, although the number of steps varies. ${ }^{10}$

\section{Objectives of knowledge translation}

An important step in the selection of interventions for knowledge translation is the choice of specific objectives for the program of knowledge translation. This step, known as goalsetting, can contribute to effective behavioural change. ${ }^{11,12}$ The objectives should be related to outcomes for patients, populations and society. For instance, the objectives for improving treatment of heart failure could include higher survival rates (e.g., resulting from better use of ACE inhibitors and $\beta$-blockers) and lower costs for health care (e.g., resulting from fewer hospital admissions). Many objectives for knowledge translation have been defined in terms of specific changes in treatment or other aspects of the delivery of health care (e.g., more prescribing of ACE inhibitors and $\beta$-blockers). The expectation is that such changes result in better outcomes. Ideally, strong evidence would support this expectation, but in reality, such evidence is not always available.

Several methods can be used to select objectives for knowledge translation (e.g., a Delphi procedure). ${ }^{13}$ A study showed, for instance, that about $30 \%$ of children seen in primary care with diagnosed urinary tract infection had not received antibiotics. ${ }^{14}$ The researchers invited nine family physicians to consider what aspects of the primary care of these patients needed to be targeted in a program of knowledge translation. A Delphi procedure was used. In the first round, family physicians received a written questionnaire about 22 potential objectives and were asked to comment on and rate the clinical relevance of these objectives.

In the second round, the researchers reported the results of the first round and offered a number of revised objectives based on the feedback from round one. This procedure resulted in seven objectives, including "all children aged younger than six months with a (suspected) urinary tract infection are referred to secondary care for treatment" and "all children with a urinary tract infection have to receive follow-up contact, during which the patient's urine is tested by dipstick or urine culture, within three to five days after finishing the antibiotic treatment." In our example involving heart failure, we could ask family physicians to rate the clinical relevance of objectives derived from national guidelines for the care of these patients.

\section{Indicators used to measure implementation}

The objectives need to be defined with specific indicators that can be used to measure the degree of implementation. Clinical guidelines or other recommended practices can be analyzed to identify such indicators. The indicators should have good measurement-related properties, support from key stakeholders and high feasibility. As outlined in a previous article, ${ }^{15}$ current best practice is a structured Delphi procedure with panels of stakeholders who review available evidence, followed by a test of the validity and reliability of the indicators in real practice settings. ${ }^{10}$

For example, a European project on management of cardiovascular risk in primary care used a two-stage Delphi procedure to select indicators. ${ }^{16}$ One hundred and one family physicians from nine countries were involved in both rounds of this procedure. From an initial list of 650 indicators, 202 indicators were derived, of which 44 were rated as valid (22\%). These indicators covered lifestyle, clinical performance and organizational aspects of care. Different instruments were developed for the measurement of these indicators. In our example involving heart failure, we could use our practice guidelines to identify potential indicators and then use a Delphi process to ask family physicians to select those that they believe are evidence-based, feasible and clinically important.

\section{Potential barriers to change}

Most planning models suggest analyzing each chosen objective with respect to barriers to change. For instance, reducing the inappropriate use of proton pump inhibitors may be hampered by patients' resistance to changing their routines, lack of knowledge on the part of physicians (e.g., about the adverse effects of these drugs), organizational routines such as automatic delivery of repeat prescriptions, and financial incentives (e.g., more prescriptions translate into higher income for pharmacies). ${ }^{17}$ Analysis of each objective in detail is usually not possible, so objectives have to be prioritized.

A wide range of methods exists for the identification of barriers to change ${ }^{10}$ and was discussed in more detail in an earlier paper in this series. ${ }^{1}$ Briefly, these methods can be divided into three broad categories. A first category comprises methods (i.e., interviews, questionnaires and group-based methods) to identify barriers to change as reported by professionals, patients and others. This type of method can be used simply or more scientifically, but its disadvantage is that the barriers reported may have little or no impact on knowledge translation in reality. An example is the study on barriers to changing treatment of heart failure (described above), which was based on semistructured questionnaires. ${ }^{6}$ This study found that family physicians perceived, on average, four barriers in prescribing ACE inhibitors or optimizing the dosage of these drugs. However, no significant relationships were found between the 
barriers they perceived and the prescribing of ACE inhibitors.

The other two approaches are based on quantitative analysis of available data. A second category comprises the analysis of variation in practice with respect to its determinants. This approach requires large sets of observational data on variation in the delivery of health care across patients. The study of variation in the treatment of heart failure as a result of comorbidities or use of other medications was a good example of this approach. ${ }^{5}$ A third category of methods involves the analysis of determinants of change after application of interventions for knowledge translation. This approach requires longitudinal data. An example is a meta-regression analysis of studies of the implementation of guidelines in hospital settings, which found some evidence for the influence of organizational factors on the effectiveness of interventions for knowledge translation. ${ }^{18}$

\section{Linking interventions to these barriers}

Once barriers to change have been identified, the next step is to link specific interventions to these barriers. This process is similar to the tailoring of a clinical treatment to a diagnosed health problem. ${ }^{8}$ For instance, a project that aimed to reduce inappropriate long-term use of proton pump inhibitors in patients with dyspepsia focused on one specific barrier. This barrier was described as the routine provision of repeat prescriptions without evaluating its effectiveness or discussing its usefulness with the patient. We developed and successfully tested a letter of discontinuation for patients. ${ }^{19}$ Another study found that some patients with nonspecific low back pain resisted advice to stay physically active and avoid passive physiotherapy. We developed and successfully tested a training session for physicians that included training in communication skills. ${ }^{20}$

In our example involving heart failure, we developed outreach-based visits to family physicians with educational and organizational components. A trained nonphysician visitor helped to identify patients with chronic heart failure among the patients registered with the practice and encouraged the physician to involve the practice nurse more actively in the care of patients with chronic heart failure. Furthermore, she provided a written decision tree on pharmaceutical and nonpharmaceutical therapy of chronic heart failure that was based on prevailing practice guidelines for primary care of patients with chronic heart failure. A family physician with specific expertise in chronic heart failure was available by telephone for further questions.

Linking interventions for knowledge translation to barriers is difficult because providing clear guidance on how to proceed is challenging. Both exploratory and theory-inspired methods can be used. Exploratory methods are aimed at avoiding implicit assumptions about what would work, and instead advocate using an "open mind." Group-based brainstorming is used to identify as many solutions as possible to a problem. ${ }^{21}$ For instance, a project was aimed at developing a lifestyle-specific program for patients with leg ulcers who visited dermatology departments of hospitals. A core feature of the program was the availability of a specialized nurse who had a counselling-based role and helped the patients identify options for improvements in their lifestyles. The researchers used group-based methods for the development of the program. ${ }^{22}$ In our experience, the type of implementation-specific interventions suggested by participants can be anticipated. They tend to mention what they know, such as continuing professional education and solutions based on information technology. Involvement of a wide range of stakeholders in this process could contribute to the success of the knowledge translation program.

Alternatively, theory is used to understand the factors that determine variation and change in practice. ${ }^{23,24}$ For instance, indepth interviews of general practitioners in England were used to identify obstacles to implementing guidelines for depression. ${ }^{25}$ For every comment that related to obstacles to change, a psychological theory explaining aspects of behavioural change in individuals was suggested by the team of researchers. The theory was then used to select the method of implementation.

A "common sense" use of theories would involve considering the chosen objectives and deciding which interventions are suggested by the theories to influence the determinants for change. This decision can be made in a group, so that the method used is close to the exploratory method. Suggestions about which interventions for knowledge translation could be linked to theory-based factors are provided in Appendix 1 (available at www.cmaj.ca/cgi/content/full/cmaj.081233/DC1). No firm research-based evidence exists to support either exploratory or theory-based approaches to creating an intervention for knowledge translation. We suggest combining explorative and theory-based methods to select interventions. Explorative methods may help in the consideration of issues that were not anticipated. The use of theory might help to broaden the scope of factors considered and thus reduce the chance of overlooking important issues.

\section{Factors to consider when using single or multicomponent interventions}

Although early research suggested that multicomponent interventions for knowledge translation are most effective, ${ }^{26}$ later research has raised doubts about this claim. ${ }^{7}$ Multicomponent interventions were assumed to address a larger number of barriers to change and therefore to be more effective. However, research did not clearly support this claim. The difficulty of defining what constitutes a single intervention is a complicating factor. For instance, outreach-based visits that include instruction, motivation, planning for improvement and practical help (e.g., in our scenario involving heart failure) hardly comprise a single intervention. We suggest that multicomponent interventions could be more effective than single interventions if they address different types of barriers to change.

\section{Gaps in knowledge}

How comprehensive and systematic the analysis of determinants of change has to be is not clear. The added value of tailoring interventions for knowledge translation has not been proven. A systematic review on the effectiveness of tailored 
versus nontailored interventions did not show the added value of tailoring interventions to identified barriers. ${ }^{27}$ An explorative review that included some of the same studies ${ }^{28}$ found that many interventions for knowledge translation focused on a few cognitive factors specific to clinicians, such as gaps in knowledge, although a much wider range of barriers to change were considered in the studies of determinants of change.

Many projects related to knowledge translation are pragmatic activities and should be aimed at delivering an optimal effect at the lowest possible cost. Interventions for knowledge translation should not be aimed just at improving the delivery of health care, but also at sustaining improvements. Practitioners and managers have every reason to be critical about resource-consuming methods. More research is needed on the linkage between barriers to change and the choice of interventions related to knowledge translation. Defining testable hypotheses is a challenge for researchers, even in situations that are, to some extent, unique, and in complex programs of knowledge translation that address multiple issues and stakeholders. Research showing a sustained impact of interventions for knowledge translation is also needed. ${ }^{29,30}$

Specific areas requiring research include the effectiveness and efficiency of systematic development of interventions for knowledge translation compared with pragmatic, simple methods for choosing interventions. Another issue for study is how different stakeholders, including patients, are best involved in the development of interventions for knowledge translation. Continued research is needed on determinants of improvement in health care, so that knowledge on such determinants can guide the choice of interventions for knowledge translation.

This article has been peer reviewed.

Competing interests: None declared.

Contributors: All of the authors were involved in the conception and development of the article. Michel Wensing drafted the article, and Marije Bosch and Richard Grol critically revised it for important intellectual content. All of the authors approved the final version submitted for publication.

\section{REFERENCES}

1. Harrison MB, Légaré F, Graham ID. Adapting clinical practice guidelines to local context and assessing barriers to their use. CMAJ 2009 Dec. 7 [Epub].

2. Graham ID, Logan J, Harrison MB, et al. Lost in knowledge translation: time for a map? J Contin Educ Health Prof 2006;26:13-24.

3. Cleland JG, Cohen-Solal A, Aguilar JC, et al. Management of heart failure in primary care (the IMPROVEMENT of Heart Failure Program): an international survey. Lancet 2002;360:1631-9.

4. Sturm HB, Van Gilst WH, Swedberg K, et al. Heart failure guidelines and prescribing in primary care across Europe. BMC Health Serv Res 2005;5:57.

5. Sturm HB, Haaijer-Ruskamp FM, Veeger NJ, et al. The relevance of comorbidities for heart failure treatment in primary care: a European study. Eur J Heart Fail 2006;8:31-7.

6. Kasje WN, Denig P, De Graeff PA, et al. Perceived barriers for treatment of chronic heart failure in general practice: are they affecting performance? $B M C$ Fam Pract 2005;6:19.

7. Grimshaw J, Thomas RE, Maclennan G, et al. Effectiveness and efficiency of guideline dissemination and implementation strategies. Health Technol Assess 2004:8:1-72.

8. Grol RP, Grimshaw J. From best evidence to best practice: effective implementation of change in patients' care. Lancet 2003;362:1225-30.

9. Grol RP, Bosch MC, Hulscher ME, et al. Planning and studying improvement in patient care: the use of theoretical perspectives. Milbank $Q$ 2007;85:93-138

10. Grol R, Wensing M, Eccles M. Improving patient care the implementation of change in clinical practice. Philadelphia (PA): Elsevier; 2004.

11. Graham ID, Tetroe J.; KT Theories Research Group. Some theoretical underpinnings of knowledge translation. Acad Emerg Med 2007;14:936-41.

12. Locke EA, Latham GP. A theory of goal setting and task performance. Englewood Cliffs (NJ): Prentice Hall; 1991.
13. Linstone HA, Turoff M. The Delphi method: techniques and applications. Reading (MA): Addison-Wesley; 1975.

14. Harmsen M, Wensing M, Braspenning JCC, et al. Management of children's urinary tract infections in Dutch family practice: a cohort study. BMC Fam Pract 2007;13:8-9.

15. Kitson A, Straus SE. The knowledge-to-action cycle: identifying the gaps. CMAJ 2009 Nov. 30 [Epub]

16. Campbell SM, Ludt S, Van Lieshout J, et al. Quality indicators for the prevention and management of cardiovascular disease in primary care in nine European countries. Eur J Cardiovasc Prev Rehabil 2008;15:509-15.

17. Krol N, Spies J, van Balen J, et al. Dyspepsia in general practice: medical care and its determinants. Oual Prim Care 2003;1:173-80.

18. Dijkstra R, Wensing M, Thomas R, et al. The relationship between organisational characteristics and the effects of clinical guidelines on medical performance in hospitals, a meta-analysis. BMC Health Serv Res 2006;6:53.

19. Krol N, Wensing M, Haaijer-Ruskamp F, et al. Patient-directed strategy to reduce prescribing for patients with dyspepsia in general practice: a randomized trial. Aliment Pharmacol Ther 2004;19:917-22.

20. Engers AJ, Wensing M, van Tulder MW, et al. Implementation of the Dutch low back pain guideline for general practitioners: a randomized controlled trial. Spine 2005;30:559-600.

21. Osborn AF. Applied imagination: principles and procedures of creative problem solving. 3rd ed. New York (NY): Charles Scribner's Sons; 1963.

22. Heinen MM, Bartholomew LK, Wensing M, et al. Supporting adherence and healthy lifestyles in leg ulcer patients: systematic development of the Lively Legs program for dermatology outpatient clinics. Patient Educ Couns 2006;61:279-91.

23. Eccles M, Grimshaw J, Walker A, et al. Changing the behaviour of healthcare professionals: the use of theory in promoting the uptake of research findings. J Clin Epidemiol 2005;58:107-12.

24. Sales A, Smith J, Curran G, et al. Models, strategies, and tools. Theory in implementing evidence-based findings in health care practice. J Gen Intern Med 2006;21:S43-9.

25. Baker R, Reddish S, Robertson N, et al. Randomized controlled trial of tailored strategies to implement guidelines for the management of patients with depression in general practice. Br J Gen Pract 2001;51:737-41.

26. Wensing M, Van der Weijden T, Grol R. Implementing guidelines and innovations in primary care: which interventions are effective? Br J Gen Pract 1998;48:991-7.

27. Shaw B, Cheater F, Baker R, et al. Tailored interventions to overcome identified barriers to change: effects on professional practice and health outcomes [review]. Cochrane Database Syst Rev 2005;(1):CD005470.

28. Bosch M, Van der Weijden T, Wensing M, et al. Tailoring quality improvement interventions to identified barriers: a multiple case analysis. J Eval Clin Pract 2007; 13:161-8.

29. Grol R, Berwick DM, Wensing M. On the trail of quality and safety in health care BMJ 2008:336:74-6.

30. Tetroe JM, Graham ID, Foy R, et al. Health research funding agencies' support and promotion of knowledge translation: an international study. Milbank $Q 2008$; 86:125-55.

Correspondence to: Michel Wensing, Scientific Institute for Quality of Healthcare, Radboud University Nijmegen Medical Centre, PO Box 9101, 6500 HB Nijmegen, Netherlands; m.wensing@iq.umcn.nl

\section{Articles to date in this series}

- Straus SE, Tetroe J, Graham ID. Defining knowledge translation. www.cmaj.ca/cgi/doi/10.1503/cmaj.081229

- Brouwers M, Stacey D, O'Connor A. Knowledge creation: synthesis, tools and products. www.cmaj.ca/cgi/doi/10.1503 /cmaj.081230

- Kitson A, Straus SE. The knowledge-to-action cycle: identifying the gaps. www.cmaj.ca/cgi/doi/10.1503/cmaj.081231

- Harrison MB, Légaré F. Adapting clinical practice guidelines to local context and assessing barriers to their use. www.cmaj.ca /cgi/doi/10.1503/cmaj.081232

The book Knowledge Translation in Health Care: Moving from Evidence to Practice, edited by Sharon Straus, Jacqueline Tetroe and Ian D. Graham and published by Wiley-Blackwell in 2009 , includes the topics addressed in this series. 